\title{
Distortion and quality measures for validating and generating high-order tetrahedral meshes
}

\author{
Abel Gargallo-Peiró · Xevi Roca · Jaime \\ Peraire · Josep Sarrate
}

\begin{abstract}
A procedure to quantify the distortion (quality) of a high-order mesh composed of curved tetrahedral elements is presented. The proposed technique has two main applications. First, it can be used to check the validity and quality of a high-order tetrahedral mesh. Second, it allows the generation of curved meshes composed of valid and high-quality high-order tetrahedral elements. To this end, we describe a method to smooth and untangle high-order tetrahedral meshes simultaneously by minimizing the proposed mesh distortion. Moreover, we present a $p$-continuation procedure to improve the initial configuration of a high-order mesh for the optimization process. Finally, we present several results to illustrate the two main applications of the proposed technique.
\end{abstract}

\section{Introduction}

In the last two decades, high-order methods have attracted a remarkable attention from the computational methods community. This attention has been prompted by the potential of high-order methods to deliver higher accuracy with a lower computational cost than low order methods [47, 5, 4, 26, 22, 19, 27]. However, their adoption has been hampered by several technical issues such as the difficulty of developing robust implementations and the generation of 3D curved meshes. Note that a mesh for high-order methods is composed of straight-sided and potentially curved elements. The curved elements are required to approximate adequately the curved domain boundaries and therefore, to preserve the accuracy of highorder methods $[2,7,29,49,42]$. Hence, it is mandatory to develop techniques that

A preliminary short version of this work appeared in the Proceedings of the 2013 International Meshing Roundtable [13].

Abel Gargallo-Peiró and Josep Sarrate Laboratori de Càlcul Numèric (LaCàN),

Universitat Politècnica de Catalunya, Barcelona 08034, Spain

E-mail: abel.gargallo@upc.edu, jose.sarrate@upc.edu

Xevi Roca and Jaime Peraire

Department of Aeronautics and Astronautics

Massachusetts Institute of Technology, Cambridge, MA 02139, USA

E-mail: xeviroca@mit.edu, peraire@mit.edu 
allow the generation of valid 3D curved meshes to obtain full benefit of high-order methods.

A curved mesh is considered to be valid if each one of the elements can be transformed to a regular and straight-sided element (master) through a mapping that is differentiable, invertible and smooth (diffeomorphism). That is, the mapping between the master element and the curved element is expressed in terms of a differentiable function, and the curved element is non-folded (positive determinant of the mapping Jacobian). Moreover, a curved mesh is considered to be of high-quality if all the elements have a shape close to regular (smooth and well-conditioned Jacobian). Specifically, if an element is invalid, the determinant of the mapping Jacobian presents non-positive values. These non-positive values invalidate the change of variables used to compute the weak formulation integrals on a master element. Moreover, if an element has low-quality the Jacobian could be non-smooth or ill-conditioned. In that case, the approximation accuracy is degraded and the solution may be polluted by the introduced error [45]. Therefore, it is required to develop measures to quantify the validity and quality of a given 3D curved mesh.

Quality measures also have an alternative and significant application. They allow using optimization based techniques to repair non-valid meshes (untangle) and to improve the mesh quality (smooth) by minimizing (maximizing) the distortion (quality) of the mesh elements. Note that this technique allows the generation of high-order meshes with an a posteriori approach $[7,6,30,28,43,32]$. That is, it allows the generation of meshes that might contain inverted or low-quality elements, and then untangle and smooth them a posteriori to ensure and enhance the mesh quality. Specifically, a high-order mesh can be obtained by generating first a linear mesh. Second, the linear mesh is converted to a high-order mesh by adding additional nodes and by curving the boundary elements. Finally, the converted mesh is untangled and smoothed to remove the non-valid (folded) and low-quality (distorted) elements. However, the application of this approach together with a mesh quality optimization has been hampered by the absence of 3D quality measures for high-order elements with degree superior than two. Note that the capability of generating valid curved 3D meshes is of the major importance for the community of high-order unstructured methods.

The main contribution of this work is to present a technique that allows extending any Jacobian based quality measure for linear 3D elements to high-order simplices of any interpolation degree. In addition, we derive how to apply the proposed technique: to quantify the validity and quality of a given $3 \mathrm{D}$ curved mesh; and to generate curved meshes with an a posteriori approach based on the minimization of the mesh distortion. To illustrate these two main applications, we check the validity and quantify the quality of several curved meshes. Moreover, we present several curved meshes obtained with the presented a posteriori approach.

In this work, we also present a $p$-continuation procedure to accelerate the generation of curved high-order meshes. The main idea is to split each curved element in several linear elements determined by the high-order nodes. Then, the smoothing and untangling method is applied to this linear mesh. The resulting configuration of the nodes on this linear mesh, determines the initial configuration for the nodes on the high-order mesh. Finally, this initial configuration is smoothed and untangled to obtain the desired curved high-order mesh. 
The rest of the paper is organized as follows. In Sec. 2, we review the literature related with this work. In Sec. 3 we present the definition of distortion and quality measures for high-order simplicial elements. Next, in Sec. 4, we detail the two main applications of the proposed measures: the validation and generation of curved high-order meshes. Finally, in Sec. 5, we present several examples in order to illustrate the behavior of the defined measures and the application of the developed techniques.

\section{Related work}

One of the main issues in mesh generation is to quantify the validity of a mesh for computational purposes. For linear elements, a wide range of quality measures have been developed $[10,45]$. However, this is an unresolved issue for high-order meshes. To address this issue, we develop a technique that allows extending to high-order elements the set of Jacobian-based measures for linear elements presented in [23, 24 . These measures allow determining the quality of a linear element in terms of the Jacobian of the representation mapping. Below we review the previous developed approaches to quantify the validity and quality of curved meshes.

Different techniques have been proposed to determine the validity of a highorder mesh by means of checking the positivity of the Jacobian mapping from the master to the physical element. Specifically, it has been studied how to detect non-positive Jacobian determinants for B-spline based mappings [7,6,30, 28, $43]$ and quadratic iso-parametric elements [31,9,1]. Moreover, for higher interpolation degrees, in References [20,21] it is proposed to compute accurate bounds on Jacobian determinants of $2 \mathrm{D}$ and $3 \mathrm{D}$ curvilinear polynomial finite elements.

In contrast, several approaches have been developed to quantify the quality of non-linear iso-parametric elements. For elements of quadratic degree, different definitions of distortion (quality) have been proposed for the planar $[50,37,38,25]$ and 3D $[39,3,16]$ elements. The main difference of this work with the previous works is that we propose the definition of the distortion (quality) measure for tetrahedral elements (3D) of any interpolation degree. The proposed distortion is the $\mathcal{L}^{2}$-norm of the regularization of a given point-wise Jacobian-based measure. This definition allows the detection of non-positive values of the Jacobian determinant of the master mapping for any interpolation degree. That is, if the quality is greater than zero, the master mapping is a local diffeomorphism in the integration points. It is important to point out that this work is an extension to $3 \mathrm{D}$ of the distortion measures for planar and surface elements presented in $[34,12]$.

The defined distortion measure allows both the validation of high-order tetrahedra and the generation of high-order meshes via an a posteriori optimization approach. The a posteriori approach has been previously used with success for Bspline mappings [7,6,30,28,43], and for iso-parametric elements [44,32, 34, 48, 16 , $12,33]$. Next, we highlight the two main types of approaches to generate high-order tetrahedral meshes by means of an a posteriori approach.

On the one hand, in $[32,48]$ two techniques to generate curved meshes by means of a solid mechanics analogy are presented (non-linear and linear elasticity, respectively). On the other hand, the curving of the mesh in the a posteriori approach can also be achieved by the optimization of a distortion (quality) measure. In [16] a technique is presented to generate meshes composed of tetrahedra of degree two 
via topology regularization and optimization of two functions (scaled Jacobian measure and a ratio of the length and volume of the elements). Moreover, in [34, $12,13,14]$ we proposed to optimize the high-order nodes location according to an objective function based on the defined distortion measure for high-order meshes. In contrast, in $[33,46]$ it is proposed to optimize a function that is able to avoid Jacobians that are too small or too high.

One of the main features of the optimization approach that we propose for high-order meshes is that it allows untangling (recovering from an inverted configuration) as well as smoothing meshes of any interpolation degree. Among the reviewed optimization formulations, there are two consistent untangling techniques specialized in curving high-order meshes. Note that untangling high-order meshes is strictly necessary, since tangled elements appear in the a posteriori approach. A technique to define a regularized distortion and quality measure for high-order planar and surface elements applied to generate curved meshes is proposed in [34, 12]. The untangling capability is introduced explicitly in the formulation by means of the regularization of the Jacobian-based measure presented in [8,12]. This regularization leads to an automatic and robust smoothing-untangling optimization approach that enforces that unfeasible configurations (tangled) become valid (untangle). In contrast, in $[33,46]$ it is proposed to optimize a function that penalizes small values of the Jacobian determinants based on the parameter-dependent logbarrier method presented in $[40,41]$. In this work, we use the approach proposed in $[34,12]$. It is important to point out that the optimization process proposed here consists of a minimization of the defined high-order mesh distortion. Therefore, the resulting untangled mesh is valid, but it also maximizes the defined quality measure.

\section{Distortion and quality measures for high-order tetrahedral elements}

In this section, we first review the definition of Jacobian-based distortion measures for linear tetrahedral elements. In addition, we introduce the notation required for high-order elements. Finally, we propose a definition for a distortion and a quality measure for high-order tetrahedra in terms of the Jacobian-based distortion measures for linear elements.

\subsection{Preliminaries}

In this work, we propose to define the quality of a high-order tetrahedral element by means of a generalization of the Jacobian-based quality measures for linear elements presented in $[23,24]$. To define a Jacobian-based measure for linear tetrahedra, three elements are required: the master, the ideal, and the physical. The master element $\left(E^{M}\right)$ is the element from which the iso-parametric mapping is defined. The ideal tetrahedron $\left(E^{I}\right)$ represents the target configuration. The physical $\left(E^{P}\right)$ is the element to be measured. First, the mappings between the ideal and the physical elements through the master element are obtained. By means of these applications, a mapping between the ideal and physical elements is determined by the composition (see Figure 1)

$$
\phi_{E}: E^{I \stackrel{\phi_{I}^{-1}}{\longrightarrow}} E^{M} \stackrel{\phi_{P}}{\longrightarrow} E^{P} .
$$




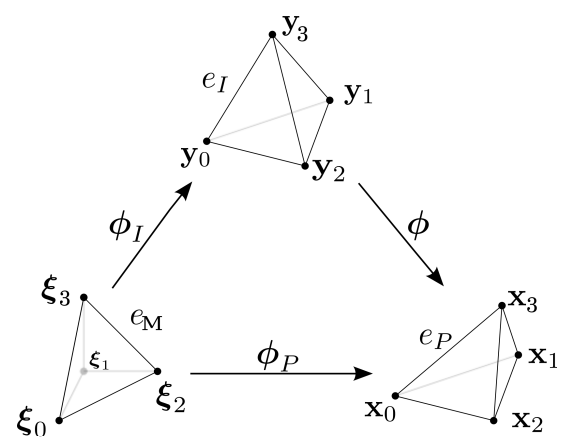

Fig. 1 Mappings between the master, ideal and physical linear elements.

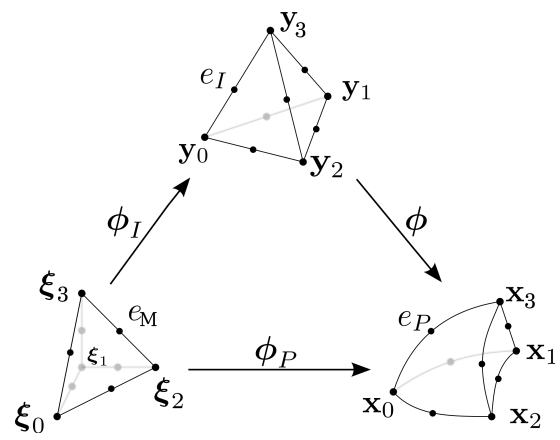

Fig. 2 Mappings between the master, ideal and physical high-order elements.

The Jacobian of this affine mapping, herein denoted by $\mathbf{D} \phi_{E}$, encodes the deviation of the physical element with respect to the ideal one. Hence, the distortion measure of the physical element is defined in terms of $\mathbf{D} \phi_{E}$. These distortion measures, herein denoted by $\eta$, quantify the deviation of one or several features (shape, size, skewness, degeneracy,...) of the physical element with respect to the ideal element in a range scale $[1, \infty)$. The corresponding quality measure is defined as $q:=1 / \eta \in[0,1]$.

For the remainder of this work, we use the shape distortion measure [23]:

$$
\eta\left(\mathbf{D} \phi_{E}\right)=\frac{\left\|\mathbf{D} \phi_{E}\right\|_{F}^{2}}{3|\sigma|^{2 / 3}}
$$

where $\|\cdot\|_{F}$ is the Frobenius norm, and $\sigma=\operatorname{det}\left(\mathbf{D} \phi_{E}\right)$. This distortion measure quantifies the deviation of the shape of the physical tetrahedron with respect to the ideal shape. To assign quality zero for degenerated elements (negative $\sigma$ ), $\sigma$ in Eq. (1) is replaced by $\sigma_{0}=(\sigma+|\sigma|) / 2$. In this way, for negative values of $\sigma$, $\eta=\infty$ and therefore, $q=0$.

To untangle meshes in the optimization procedure, we use the modification of the determinant $\sigma$ proposed in [8]. This modification can be applied to Jacobianbased distortion measures where the determinant of the Jacobian appears in the denominator. Specifically, we replace $\sigma$ in (1) by

$$
\sigma_{\delta}(\sigma)=\frac{1}{2}\left(\sigma+\sqrt{\sigma^{2}+4 \delta^{2}}\right),
$$


where $\delta$ is a numerical parameter that is determined element-wise. In our implementation, we set $\delta=0$ for valid configurations and $\delta=0.01 \cdot \sigma^{*}$ for tangled configurations, being $\sigma^{*}$ the determinant of the corresponding straight-sided ideal element, as proposed in $[15,14,11]$. Therefore, we consider a modified shape distortion measure,

$$
\eta_{\delta}\left(\mathbf{D} \phi_{E}\right)=\frac{\left\|\mathbf{D} \phi_{E}\right\|_{F}^{2}}{3\left|\sigma_{\delta}\right|^{2 / 3}} .
$$

It is important to point out that without the proposed modification, $\eta$ has an asymptote when $\sigma=0$ (where an element becomes non-valid). Thus, it is required to modify $\sigma$ to remove this asymptote and therefore, allow the optimization procedure to recover from the non-valid configuration (tangled). For $\delta>0, \sigma_{\delta}(\sigma)$ is a strictly increasing function, such that $\sigma_{\delta}(0)=\delta$ and that tends to 0 when $\sigma$ tends to $-\infty$. Therefore, $\eta_{\delta}$ is a smooth function with no asymptotes. Moreover, for small values of $\delta$, the minimum of $\eta_{\delta}$ is close to the valid minimum of $\eta_{0}$.

\subsection{Notation for iso-parametric high-order tetrahedra}

Let $E^{P}$ be a nodal high-order tetrahedron of interpolation degree $p$ determined by $n_{p}=(p+1) \cdot(p+2) \cdot(p+3) / 6$ nodes with coordinates $\mathbf{x}_{i} \in \mathbb{R}^{3}$, for $i=1, \ldots, n_{p}$. We select a node distribution that provides a quasi-optimal Lebesgue constant [17]. Given a master element $E^{M}$ with nodes $\boldsymbol{\xi}_{i} \in \mathbb{R}^{3}$, being $i=1, \ldots, n_{p}$, we consider the basis $\left\{N_{i}\right\}_{i=1, \ldots, n_{p}}$ of nodal shape functions (Lagrange interpolation) of degree $p$. Then, the high-order isoparametric mapping from $E^{M}$ to $E^{P}$ can be expressed as:

$$
\begin{aligned}
\phi_{P}: E^{M} \subset \mathbb{R}^{3} & \longrightarrow E^{P} \subset \mathbb{R}^{3} \\
\boldsymbol{\xi} & \longmapsto \mathbf{x}=\phi_{P}(\boldsymbol{\xi})=\sum_{i=1}^{n_{p}} \mathbf{x}_{i} N_{i}(\boldsymbol{\xi}) .
\end{aligned}
$$

The Jacobian of the isoparametric mapping (4) is the matrix

$$
\mathbf{D} \phi_{P}(\boldsymbol{\xi})=\sum_{i=1}^{n_{p}} \mathbf{x}_{i} \boldsymbol{\nabla} N_{i}(\boldsymbol{\xi}) \in \mathbb{R}^{3} \times \mathbb{R}^{3} .
$$

\subsection{Definition of distortion and quality}

In this section, we define the distortion and quality measure for a high-order element in terms of a Jacobian-based distortion measure for linear elements. To this end, analogously to the linear case, we consider three elements: the master $E^{M}$, the ideal $E^{I}$, and the physical $E^{P}$. The master element allows the characterization of the ideal and physical elements in terms of a master mapping. In particular, it is required to determine a distribution of points on the master element and the corresponding coordinates of the nodes in the ideal and physical elements. On the one hand, the coordinates of the nodes of the ideal element (see Sec. 4.1 for more details) are chosen to determine a straight-sided high-order tetrahedron. Therefore, $\phi_{I}$ is an affine mapping. On the other hand, the coordinates of the nodes 
of the physical element determine, in general terms, a curved element. Hence, the mapping $\phi_{P}$ presented in Eq. (4), is not affine in general, see Figure 2. Moreover, $\phi_{E}=\phi_{P} \circ \phi_{I}^{-1}$ is also not affine, and the Jacobian matrix is not constant. Specifically, for a point $\mathbf{y}$ on the ideal element, the expression of the Jacobian is:

$$
\mathbf{D} \phi_{E}(\mathbf{y})=\mathbf{D} \phi_{P}\left(\phi_{I}^{-1}(\mathbf{y})\right) \cdot \mathbf{D} \phi_{I}^{-1}(\mathbf{y})
$$

Similar to the linear case, we want to define a distortion measure based on the Jacobian matrix of $\phi_{E}$. However, the Jacobian of a high-order element is not constant. Nevertheless, the Jacobian on a point allows measuring the local deviation between the ideal and the physical element. Thus, we can obtain an elemental distortion measure by considering the $\mathcal{L}^{2}$-norm of the Jacobian-based distortion measure on the ideal element.

We define the ideal mesh $\mathcal{M}_{I}$ of a mesh $\mathcal{M}$ as the set of ideal elements that correspond to each physical element. Next, we define the scalar product of two scalar functions on $\mathcal{M}_{I}$ as

$$
\langle f, g\rangle_{\mathcal{M}_{I}}:=\sum_{i=1}^{n_{E}}\langle f, g\rangle_{E_{i}^{I}},
$$

expressed in terms of their inner product on the ideal element,

$$
\langle f, g\rangle_{E^{I}}:=\int_{E^{I}} f(\mathbf{y}) g(\mathbf{y}) \mathrm{d} \mathbf{y}
$$

where $E_{i}^{I}$ is the ideal of element $E_{i}^{P}$, and $n_{E}$ is the number of elements of the mesh. The norms corresponding to these scalar products are

$$
\begin{aligned}
\|f\|_{\mathcal{M}_{I}} & :=\sqrt{\langle f, f\rangle_{\mathcal{M}_{I}}}, \\
\|f\|_{E^{I}} & :=\sqrt{\langle f, f\rangle_{E^{I}}},
\end{aligned}
$$

Remark 1 We choose $E^{I}$ as a valid straight-sided tetrahedron. That is, $\phi_{I}$ is an affine mapping and therefore, a global diffeomorphism. In this way, we can use the change of variable determined by $\phi_{I}$ to compute the scalar product as:

$$
\langle f, g\rangle_{E}:=\int_{E^{M}} f\left(\boldsymbol{\phi}_{I}(\boldsymbol{\xi})\right) g\left(\boldsymbol{\phi}_{I}(\boldsymbol{\xi})\right)\left|\operatorname{det} \mathbf{D} \boldsymbol{\phi}_{I}(\boldsymbol{\xi})\right| \mathrm{d} \boldsymbol{\xi} .
$$

To compute this integral, we use a numerical quadrature scheme that ensures that polynomials of degree $(d+3) p-d$ and $d$ variables are integrated exactly, where $d=3$ is the number of spatial dimensions. Specifically, the quadrature use $((d+3) p-d-1) / 2$ integration points as specified in [18].

Next, we define the distortion at a point $\mathbf{y} \in E^{I}$ for a high-order element with nodes $\mathbf{x}_{1}, \ldots, \mathbf{x}_{n_{p}} \in \mathbb{R}^{3}$, as:

$$
\mathrm{M} \phi_{E}:=\eta_{\delta}\left(\mathbf{D} \phi_{E}\right)
$$

We point out that $\mathrm{M} \phi_{E}$ is a function of $\mathbf{y}$, and that it also depends on $\mathbf{x}_{1}, \ldots, \mathbf{x}_{n_{p}}$, since $\phi_{E}$ does. Note that the point-wise distortion, Eq. (12), can be defined using any distortion measure for linear elements that is expressed explicitly in terms of 
of the Jacobian (Jacobian-based) or that can be casted to an expression in terms of the Jacobian.

Now, we can define the corresponding distortion and quality measures for a high-order element:

Definition 1 The distortion measure for a high-order $3 D$ element is

$$
\eta_{E}:=\frac{\left\|\mathrm{M} \phi_{E}\right\|_{E^{I}}}{\|1\|_{E^{I}}}
$$

where $\eta_{E}$ is a function of the element nodes $\mathbf{x}_{1}, \ldots, \mathbf{x}_{n_{p}}$, since $\mathrm{M} \phi_{E}$ is. Note that $\|1\|_{E^{I}}$ is the area of the ideal element.

Remark 2 Selecting $\delta=0$ in the point-wise distortion measure for high-order elements Eq. (12), implies that the regularization introduced in Eq. (2) is null, and the point-wise distortion is divergent. Therefore, if an element is inverted in a region of non-null measure, the $\mathcal{L}^{2}$-norm of the point-wise distortion in Eq. (12) will be infinite.

Definition 2 The quality measure for a high-order planar element is $q_{E}:=1 / \eta_{E}$.

\section{Application to meshing}

In this section, we analyse the two main applications of the defined distortion and quality measures. First, we overview the main features that allow using these measures to validate a given high-order tetrahedral mesh. Second, we set an optimization framework in order to generate high-order tetrahedral meshes in an $a$ posteriori procedure.

\subsection{Validation of high-order tetrahedral meshes}

One of the main applications of distortion (quality) measures is to check that the mesh is valid to perform a numerical simulation. Specifically, a quality measure has to properly detect if an element it is non-valid (and assign 0 value). Moreover, the measure has to penalize the deviation of the element with respect to the target ideal (and assign value 1 to the ideal).

To measure the validity of elements, $\delta$ is set to 0 in (12). Therefore, if there is a point $\mathbf{y}$ where the Jacobian is non-positive $(\sigma \leq 0)$, then $\operatorname{M} \phi_{E}(\mathbf{y})=\infty$. Hence, $\eta_{E}=\infty$. Conversely, if the physical element is the ideal, $\phi_{E}$ is the identity. Then, the point-wise distortion $\mathrm{M} \phi_{E}(\mathbf{y})$ is 1 for all $\mathbf{y} \in E^{I}$. Thus, by Definition 1 , the element distortion $\eta_{E}$ is also 1 . Summarizing, we state the following remark:

Remark 3 The distortion measure $\eta_{E}$ for high-order tetrahedral elements has image $[1, \infty)$, where 1 corresponds to the ideal configuration and $\infty$ to a non-valid one. Hence, by Definition $2, q_{E}$ has image [0,1], where 0 corresponds to an inverted element, and 1 to the ideal one. 
The measures defined in Sec. 3.3 can accommodate different ideal elements. We select two different type of ideals depending on the framework of the computation. On the one hand, for absolute quality measurement, we select the equilateral tetrahedron as ideal (standard ideal for isotropic meshes [23]). The equilateral tetrahedron is used to obtain an absolute value of the quality, where all the elements are measured with respect the same target tetrahedron.

On the other hand, for optimization purposes we use a relative quality measurement. That is, we assume that we have an initial linear mesh, and for each high-order curved element we select the corresponding initial straight-sided element as ideal. It is important to point out that we assume that the initial linear mesh verifies the geometrical constraints for the numerical simulation, see Sec. 1. Therefore, in the a posteriori mesh generation procedure, our final goal is to optimize the location of the inner nodes to obtain a valid (without tangled elements) high-order mesh composed of elements with a shape similar to the one in the initial linear mesh.

\subsection{Generation of curved and high-order tetrahedral meshes}

The second application for the defined distortion measures is the generation of high-order meshes via an a posteriori approach [32]. First, we present the main steps of the a posteriori approach. Specifically, we derive an optimization method based on the minimization of the presented distortion measure. This procedure will be used to curve the high-order mesh so that the invalid boundary elements become valid. Second, we detail a $p$-continuation method to determine an initial configuration of the high-order mesh that improves the initial high-order distribution and therefore, reduces the iterations required in the optimization of the high-order mesh.

\subsubsection{A posteriori mesh generation}

The a posteriori mesh generation approach is composed of several steps. First, we generate a linear tetrahedral mesh $\left(\mathcal{M}_{I}\right)$. The elements of this mesh have the shape and the size that hold the requirements for the desired numerical computation. Second, we increase the degree of the elements and curve the boundary faces to fit the CAD geometry. For each high-order element, we set as ideal the corresponding straight-sided element in the linear mesh $\mathcal{M}_{I}$. Note that the boundary elements can be inverted or present low quality. Finally, we smooth and untangle the highorder curved mesh by minimizing the distortion of the curved mesh with respect to the straight-sided ideal.

It is important to point out that the surface mesh itself can be tangled when the faces are curved to fit the geometry. If there is a non-valid surface element, the 3D tetrahedron cannot recover from the inversion. Therefore, the a posteriori approach that we use for 3D high-order meshes is composed of two optimization steps. First, we smooth and untangle the surface mesh [12]. Second, once the surface mesh is valid, we fix the nodes of the boundary surfaces and then we optimize the volume mesh. In this section, we present an algorithm to optimize the distortion of high-order tetrahedral meshes in order to obtain valid final meshes. 
The main goal of a simultaneous smoothing and untangling method is to obtain high-quality meshes composed of valid (non-inverted) elements. The best possible result, can be characterized in terms of the distortion measure. That is, given a distortion measure $\eta$ and a mesh $\mathcal{M}$ composed of $n_{N}$ nodes and $n_{E}$ elements, the node location is ideal if

$$
\mathrm{M} \phi_{E}=1, \quad \forall \mathbf{y} \in \mathcal{M}_{I}, \quad \text { (ideal mesh distortion) }
$$

where $\mathrm{M} \phi_{E}$ is the point-wise distortion presented in (12), and $\mathcal{M}_{I}$ is the mesh composed of the ideal elements (initial straight-sided mesh). That is,

$$
\mathrm{M} \phi_{E}\left(\mathbf{y} ; \mathbf{x}_{j, \hat{1}}, \ldots, \mathbf{x}_{j, \hat{n}_{p}}\right)=1, \quad \forall \mathbf{y} \in E_{j}^{I}, j=1, \ldots, n_{E},
$$

where $E_{j}^{I}$ is the ideal element corresponding to the element $E_{j}$, and the pairs $(j, \hat{i})$ in $\mathbf{x}_{j, \hat{i}}$ identify the local $\hat{i}$-th node of element $j$ with their global mesh number $i$. However, for a fixed mesh topology and a given surface, the node location that leads to an ideal mesh distortion is in general not achievable. That is, the constraints in Eq. (14) cannot be imposed strongly and therefore, we enforce the ideal mesh distortion in the least-squares sense.

For a given mesh topology and a set of fixed nodes (nodes on the surface boundary), we formulate the non-linear least-squares problem in terms of the coordinates of a set of free nodes (nodes in the interior of the domain). To this end, we reorder the coordinates of the nodes, $\mathbf{x}_{i}$, in such a way that $i=1, \ldots, n_{F}$ are the indices corresponding to the free nodes, and $i=n_{F}+1, \ldots, n_{N}$ correspond to the fixed nodes. Defining

$$
f\left(\mathbf{x}_{1}, \ldots, \mathbf{x}_{n_{F}} ; \mathbf{x}_{n_{F}+1}, \ldots, \mathbf{x}_{n_{N}}\right):=\frac{1}{2}\left\|\mathrm{M} \phi_{E}-1\right\|_{\mathcal{M}_{I}}^{2},
$$

we can formulate the mesh optimization problem as

$$
\min _{\mathbf{x}_{1}, \ldots, \mathbf{x}_{n_{F}}} f\left(\mathbf{x}_{1}, \ldots, \mathbf{x}_{n_{F}} ; \mathbf{x}_{n_{F}+1}, \ldots, \mathbf{x}_{n_{N}}\right) .
$$

In this work, we solve this least-squares problem by means of a standard non-linear iterative Gauss-Seidel method. In this manner, we can exploit the local nature of the global objective function since a node only influences its neighbor elements, as we pointed out in [12].

Remark 4 The goal of the proposed method is to provide a robust smoothing and untangling procedure to improve the quality of a given high-order mesh. However, there are meshes that cannot be untangled by the proposed method, for instance, when the boundary mesh presents self-intersections. Nevertheless, the proposed method has properly smoothed and untangled all the tested meshes that present a valid boundary mesh.

\subsubsection{Initial configuration: $p$-continuation}

In this section, we detail a $p$-continuation procedure to improve the initial configuration of a high-order invalid mesh for the optimization procedure presented in Sec. 4. The proposed approach is composed of three steps. 

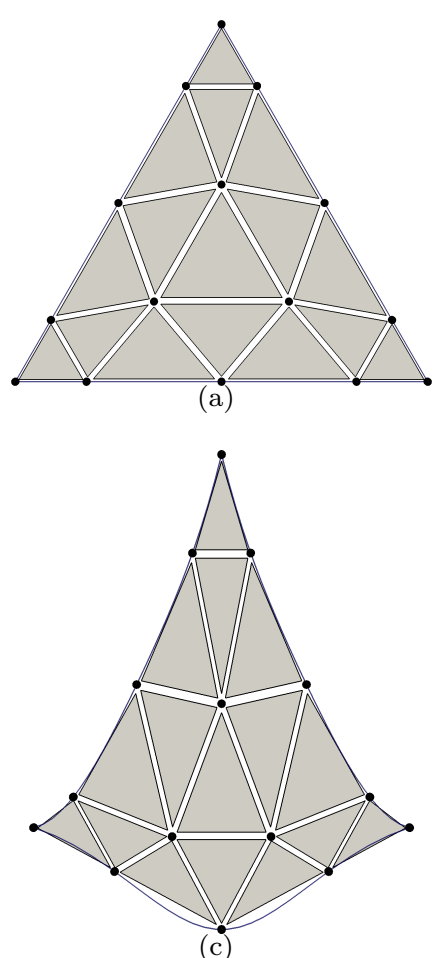

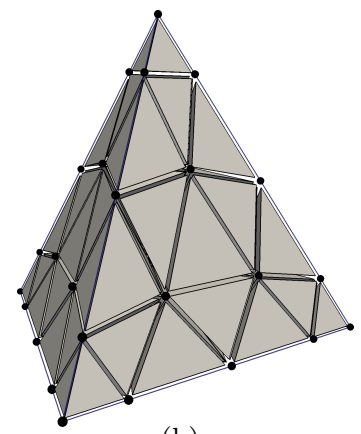

(b)

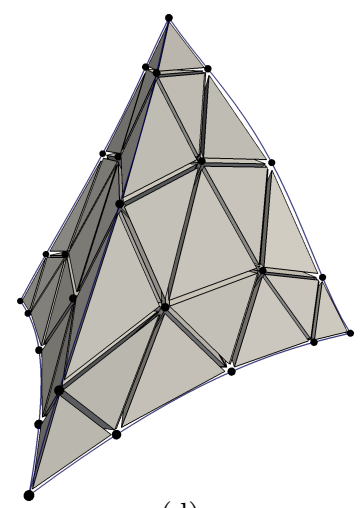

(d)

Fig. 3 Decomposition of a straight-sided $(\mathrm{a}, \mathrm{b})$ and a curved $(\mathrm{c}, \mathrm{d})$ triangle and tetrahedral elements of interpolation degree four into the corresponding linear sub-elements.

First, we generate a linear mesh by decomposing each high-order element into several structured linear elements determined by the high-order nodes. Specifically, each triangle of interpolation degree $p$ is decomposed into $\mathcal{O}\left(p^{2}\right)$ linear triangles, and each high-order tetrahedron into $\mathcal{O}\left(p^{3}\right)$ linear tetrahedra. The obtained linear mesh has the same nodes of the high-order mesh. Moreover, we assign to each linear sub-element a different ideal linear element. The ideal element associated to each sub-element is the corresponding sub-element of the ideal high-order element. In Figure 3 we show the linear decomposition of a triangle and a tetrahedron, both of interpolation degree 4 , for a straight-sided and a curved configuration.

The second step is the optimization of the linear sub-mesh using an objective function based on the distortion measure for linear elements presented in Eq. (3). Since the linear sub-mesh can also contain inverted elements, it is mandatory that the optimization method for linear elements also allows untangling invalid meshes. To meet this requirement, in this work, we use the optimization technique presented in $[8,15]$. Finally, in the third step we recover the high-order mesh by updating the locations of the nodes.

An untangled linear sub-mesh is a necessary condition to have a valid highorder mesh. Therefore, we only apply our procedure when this necessary condition does not hold, i.e. if the linear sub-mesh has invalid elements. In this context, our 


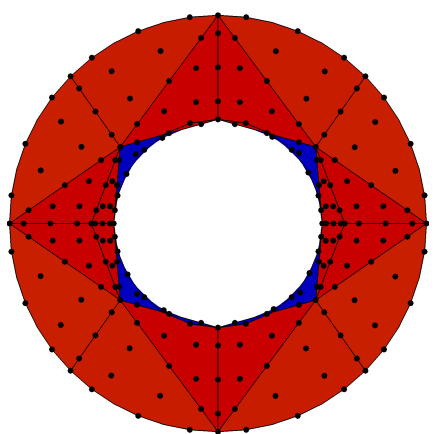

(a)

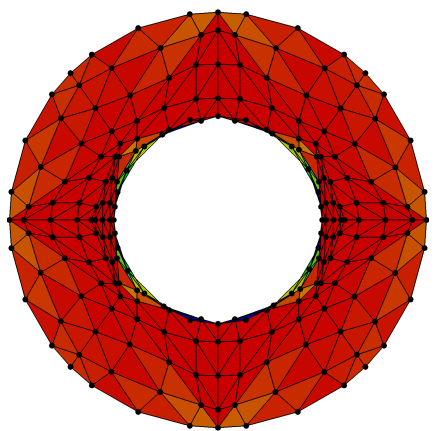

(b)

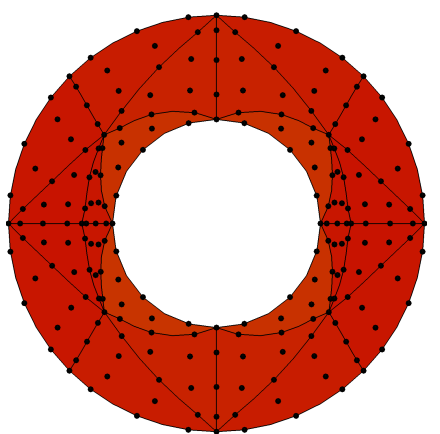

(c)

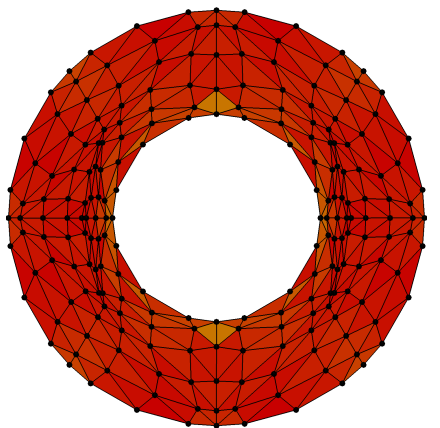

(d)

$\begin{array}{lll}0.25 & 0.5 & 0.75\end{array}$

Fig. 4 Procedure to find an initial mesh configuration. High-order meshes: (a) initial invalid configuration, (b) mesh configuration obtained with the smoothing of the linear sub-mesh. Linear sub-meshes: (c) initial invalid configuraion, (d) optimized configuration.

procedure guarantees that the quality of the initial high-order configuration is improved, since it obtains an untangled linear sub-mesh. We highlight that having a valid linear sub-mesh is a necessary, but not sufficient, condition. Hence, the updated high-order mesh can still be invalid after this procedure. This is not the case of the example presented in Figure 4, where the final high-order mesh is also untangled. However, since the validity of the high-order mesh cannot be guaranteed with this procedure, it is required to use the high-order optimization process presented in Sec. 4 to obtain a valid and high-quality high-order mesh.

To illustrate the $p$-continuation process, in Figure 4 we show a triangular mesh of interpolation degree four on a circular ring. Figure 4 (a) presents the initial highorder mesh obtained after curving the boundary edges of the high-order ideal mesh. Next, in Figure 4(b) we show the initial linear sub-mesh generated by decomposing the high-order elements. Note that the high-order mesh has four invalid elements, and the corresponding linear sub-mesh has twelve tangled triangles. Figure 4(d) displays the optimized linear sub-mesh. Notice that it does not contain inverted elements. Finally, in Figure 4(c) the high-order mesh is recovered. The quality of this mesh configuration has been improved with respect to the initial one, and 
Table 1 Time of the different optimization processes relative to the total time of the highorder optimization enhanced with the $p$-continuation technique.

\begin{tabular}{cccc} 
Approach & Technique & Rel. time & Iter. \\
\hline \multirow{2}{*}{ With $p$-continuation } & $p$-cont & 0.875 & 55 \\
& high-order & 0.125 & 6 \\
Without $p$-continuation & $p$-cont & 0 & 0 \\
& high-order & 2.21 & 82
\end{tabular}

that it does not contain any invalid elements. This high-order mesh is the input of the optimization procedure presented in Sec. 4.

For this example, the $p$-continuation procedure saves more than half of the total computational cost of the optimization process when only the high-order method is used. For the presented 2D mesh of interpolation degree four, we have checked that each global iteration of the linear mesh represents half of the cost of the highorder one. On the one hand, if we directly run the high-order method, we require 82 iterations. On the other hand, the $p$-continuation process requires 55 iterations, and the high-order optimization procedure requires just 6 more iterations. Hence, the $p$-continuation leads to an speed-up factor of 2.21 relative to the full highorder optimization. Table 1 details the time of the different optimization processes relative to the total time of the high-order optimization enhanced with the pcontinuation technique.

\section{Results}

In this section, we present two applications in order to illustrate the properties of the proposed quality measures. First, we show that the defined measures allow checking the validity of high-order tetrahedral meshes. Specifically, we analyze the validity of three meshes of degree 2, 4 and 6 on a hollow sphere. Second, we detail the main steps of the a posteriori mesh generation approach of two different CAD geometries. We generate a mesh of interpolation degree seven on a propeller, and a mesh of degree four of a Falcon aircraft. In all the examples, we have used the $p$-continuation technique presented in Sec. 4.2.2 to improve the initial mesh configuration, and next, we have optimized the obtained high-order mesh using the procedure presented in Sec. 4.2.1. The proposed algorithm has been implemented in $\mathrm{C}++$ in the meshing environment EZ4U $[35,36]$.

\subsection{Validation of curved high-order tetrahedral meshes}

In this section, we illustrate that the defined measure is capable of quantifying the validity of a high-order tetrahedron. In particular, we show that the measure detects when an element is valid or not. Moreover, it properly determines the deviation of a given element with respect to the considered ideal. Finally, we show that the presented untangling procedure is robust and can untangle high-order meshes composed of inverted elements.

We consider a hollow sphere of inner radius 1 and external radius of 3 , and we generate a coarse tetrahedral mesh. Then, we generate three meshes of interpolation degree 2, 4 and 6 with the same topology, and we tangle them, see Figures 


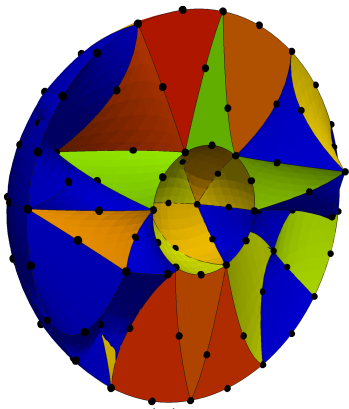

(a)

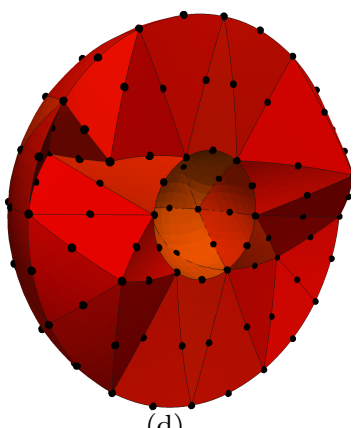

(d)

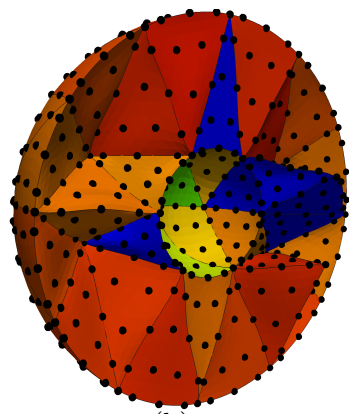

(b)

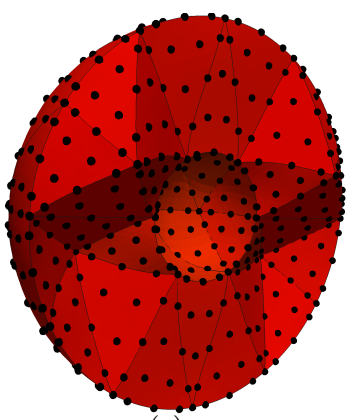

(e)

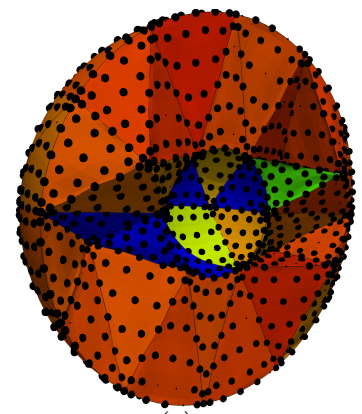

(c)

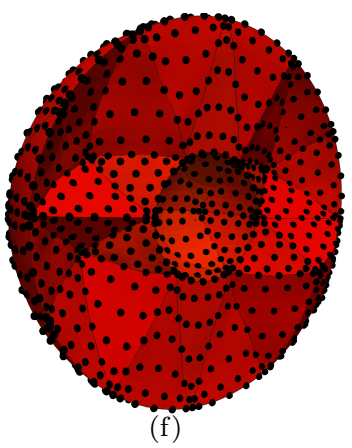

Fig. 5 Tetrahedral meshes of degree 2, 4 and 6 colored according to the shape quality measure (initial straight-sided ideal) on a hollow sphere: (a-c) initial meshes, and (d-f) smoothed meshes.

Table 2 Shape quality statistics for a hollow sphere. The mesh is composed of 160 elements.

\begin{tabular}{|c|c|c|c|c|c|c|c|c|}
\hline Degree & \#nodes & Mesh & Figure & Min. Q. & Max. Q. & Mean Q. & Std. Dev. & Tang. el. \\
\hline \multirow{2}{*}{2} & \multirow{2}{*}{302} & Initial & $5(\mathrm{a})$ & 0.00 & 0.97 & 0.51 & 0.40 & 59 \\
\hline & & Smoothed & $5(\mathrm{~d})$ & 0.91 & 0.99 & 0.98 & 0.02 & 0 \\
\hline \multirow{2}{*}{4} & \multirow{2}{*}{2042} & Initial & $5(\mathrm{~b})$ & 0.00 & 0.97 & 0.81 & 0.28 & 16 \\
\hline & & Smoothed & $5(\mathrm{e})$ & 0.95 & 1.00 & 0.99 & 0.01 & 0 \\
\hline \multirow{2}{*}{6} & \multirow{2}{*}{6502} & Initial & $5(\mathrm{c})$ & 0.00 & 0.96 & 0.81 & 0.27 & 15 \\
\hline & & Smoothed & $5(\mathrm{f})$ & 0.95 & 1.00 & 0.99 & 0.01 & 0 \\
\hline
\end{tabular}

5(a), 5(b) and 5(c). In Figures 5(d), 5(e) and 5(f) we present the smoothed meshes. Note that the optimization approach generates valid and high-quality meshes. In Table 2 we present the quality statistics of the obtained mesh. All the inverted elements have been untangled and the overall quality statistics improved. The quality statistics and the displayed quality in Figure 5 is computed taking as ideal the initial linear mesh. Therefore, we observe that in Figures 5(d), 5(e) and 5(f) almost all the elements are of quality one. That is, the optimized mesh is close to the straight-sided one. 


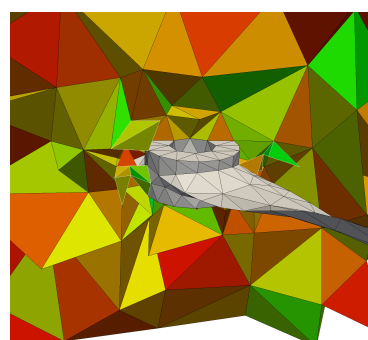

(a)

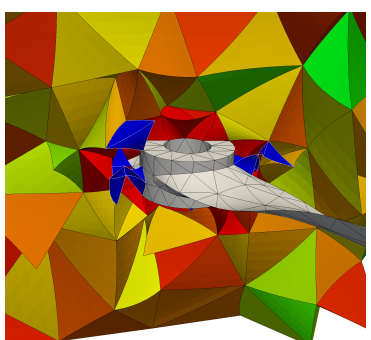

(b)

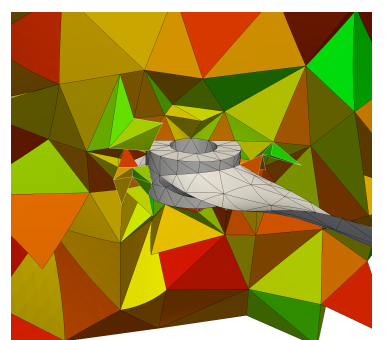

(c)

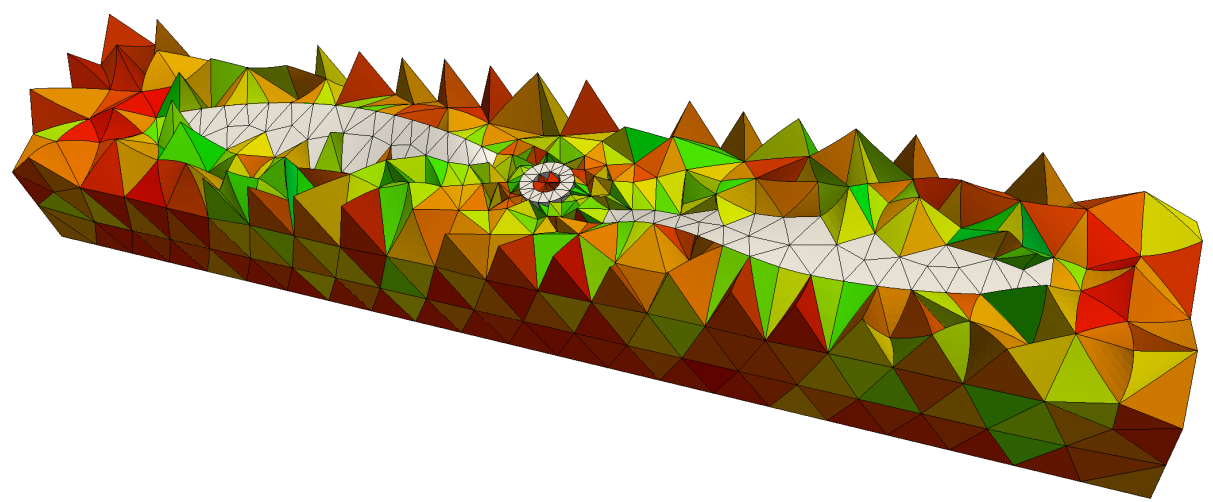

(d)

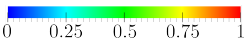

Fig. 6 Tetrahedral meshes of interpolation degree 7 colored according to the shape quality (equilateral ideal) measure for the exterior domain of a propeller: (a) initial straight-sided mesh, (b) initial curved mesh, and (c) smoothed mesh.

Table 3 Shape quality statistics of a mesh of interpolation degree 7 for the propeller. The mesh is composed of 5111 elements and 309170 nodes.

\begin{tabular}{cccccccc}
\hline Mesh & Quality & Figure & Min. Q. & Max. Q. & Mean Q. & Std. Dev. & Tang. el. \\
\hline \multirow{2}{*}{ Initial } & Absolute & $6(\mathrm{~b})$ & 0.00 & 1.00 & 0.61 & 0.22 & 72 \\
& Relative & - & 0.00 & 1.00 & 0.96 & 0.12 & 72 \\
\multirow{2}{*}{ Smoothed } & Absolute & $6(\mathrm{c})$ & 0.20 & 1.00 & 0.78 & 0.12 & 0 \\
& Relative & - & 0.78 & 1.00 & 0.99 & 0.01 & 0 \\
\hline
\end{tabular}

\subsection{Generation of curved and high-order tetrahedral meshes}

The main goal of this example is to show the a posteriori mesh generation process on two CAD geometries. Specifically, we generate a high-order mesh of degree 7 for the exterior domain of a propeller, and a mesh of degree 4 for the exterior domain of a Falcon aircraft.

First, we generate a linear mesh on both geometries. Once a linear mesh is generated, the degree of the mesh is increased. We choose these high-order straight-sided meshes as ideal, since we want to preserve the features (shape, size, isotropy...) that have been assigned to the linear meshes, see Figures 6(a) and 7(a). It is important to point out that we use the shape quality measure, presented in Eq. (3), to define the point-wise distortion measure in Eq. (13). That is, in this 


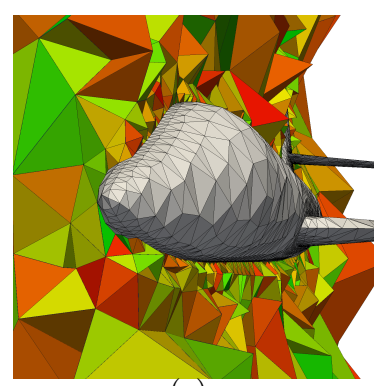

(a)

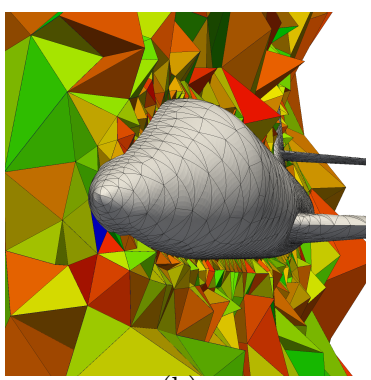

(b)

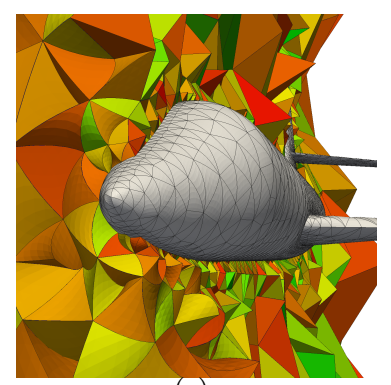

(c)

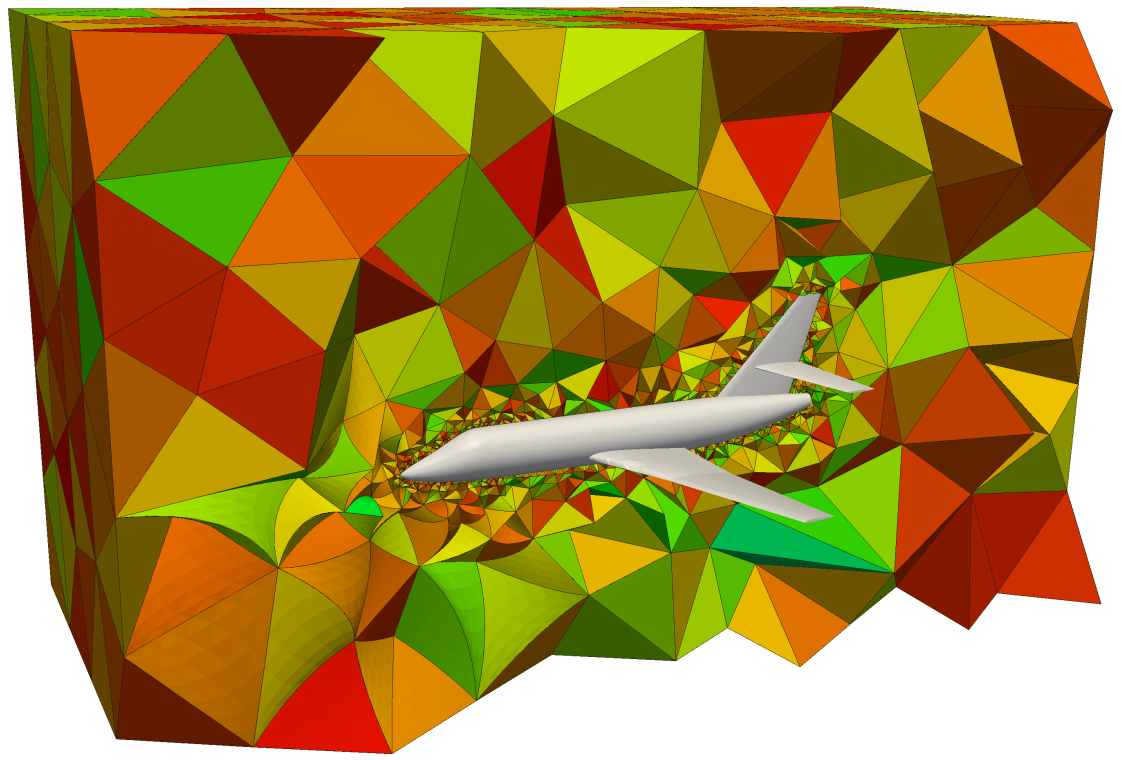

(d)

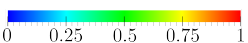

Fig. 7 Tetrahedral meshes of interpolation degree 4 colored according to the shape quality (equilateral ideal) measure for the exterior domain of a Falcon aircraft: (a) initial straight-sided mesh, (b) initial curved mesh, and (c) smoothed mesh.

work, we only impose that the shape of the initial linear mesh is preserved. In practice, this approach leads to meshes that resemble the element size of the initial linear mesh. Therefore, the element size can be imposed in practice by generating a linear mesh with the proper size distribution. To impose explicitly that the element size is preserved, it is required to use a distortion that combines at the same time a shape and size measure as we detailed in [12]. To preserve additional geometrical features of the elements other Jacobian-based distortion measures can be considered [23].

Next, the boundary faces are curved to fit the CAD representation. In Figures 6 (b) and $7(\mathrm{~b})$ we present the initial curved meshes. This process leads to several 


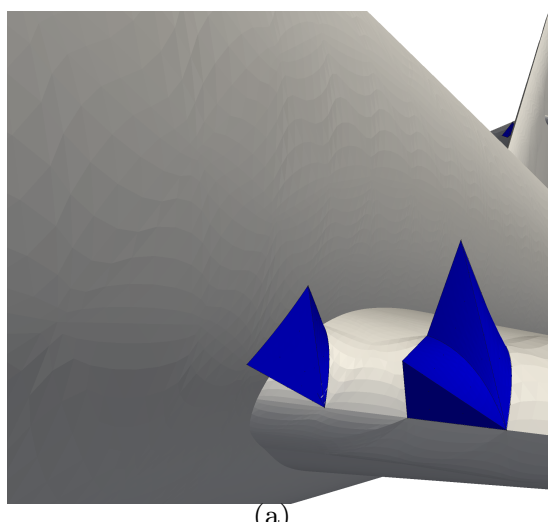

(a)

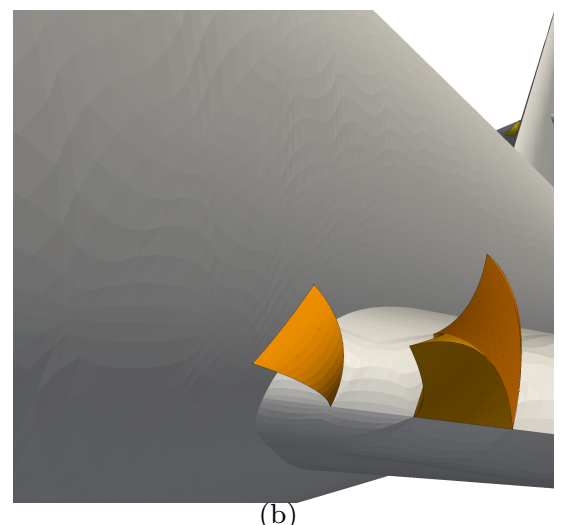

(b)

Fig. 8 Tetrahedral meshes of degree 4 colored according to the shape quality measure (equilateral ideal) for the exterior domain of a Falcon aircraft. Detail of the mesh where inverted elements can be observed: (a) initial curved mesh, and (b) smoothed mesh.

non-valid and low-quality elements, see Tables 3 and 4. In Figure 8(a), we illustrate some inverted elements that originate after curving the boundary faces on the wing of a Falcon aircraft.

Finally, we optimize the meshes. To this end, we relocate the nodes on the CAD surfaces [12], and afterwards we optimize the $3 \mathrm{D}$ meshes by means of the approach presented in Sec. 4. In Figures 6(c) and 7(c) we present the optimized meshes. Note that all the non-valid elements of the mesh have been repaired. Specifically for the Falcon aircraft, in Figure 8(b) we show the smoothed set of elements that correspond to the originally inverted ones in Figure 8(a).

It is important to point out that we evaluate the quality of the meshes with respect to two different ideals: the equilateral tetrahedron, and the initial straightsided high-order tetrahedra. The optimization is developed taking as ideal the initial straight-sided high-order mesh, since we assume that this mesh has the necessary computational requirements. However, the equilateral tetrahedron is used in isotropic meshes to allow the comparison of the qualities between different elements.

In Figure 9, we show a detail of the mesh around the left wing of the Falcon aircraft with the elements colored according to the element quality computed using two different ideal elements. On the one hand, in Figures 9(a) and 9(b) the elements are colored taking as ideal the equilateral tetrahedron. On the other hand, Figures $9(\mathrm{c})$ and $9(\mathrm{~d})$ are colored with the quality taking as ideal the initial straight-sided high-order mesh. In Figure 9(c), we observe that almost all the elements have initial quality one, since except the elements on the boundary, the rest are still straight-sided. On the contrary, the curved elements have lower quality and they can even be non-valid. In Figure 9(d), the inverted elements have been untangled, obtaining a high-quality final mesh.

Tables 3 and 4 present the quality statistics of the generated meshes. The minimum quality with respect to the straight-sided high-order mesh has been improved from 0 to 0.778 for the propeller, and from 0 to 0.32 for the Falcon 


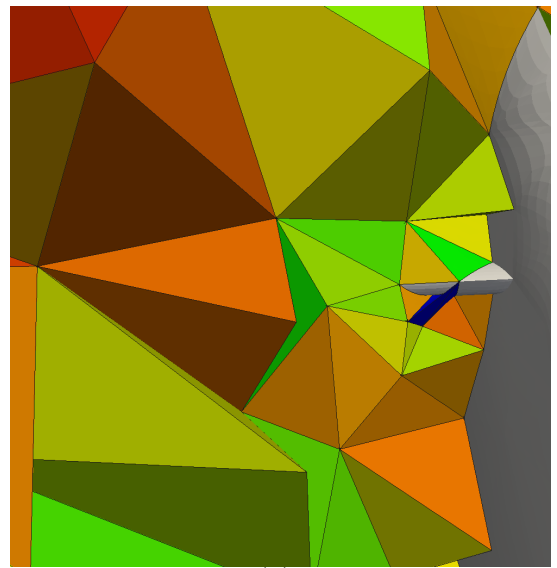

(a)

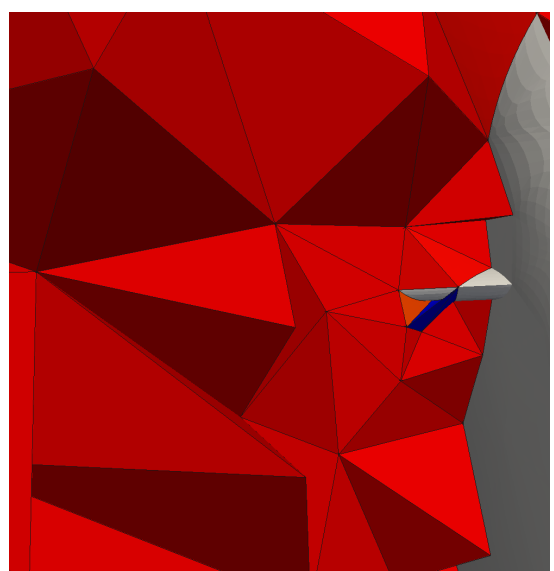

(c)

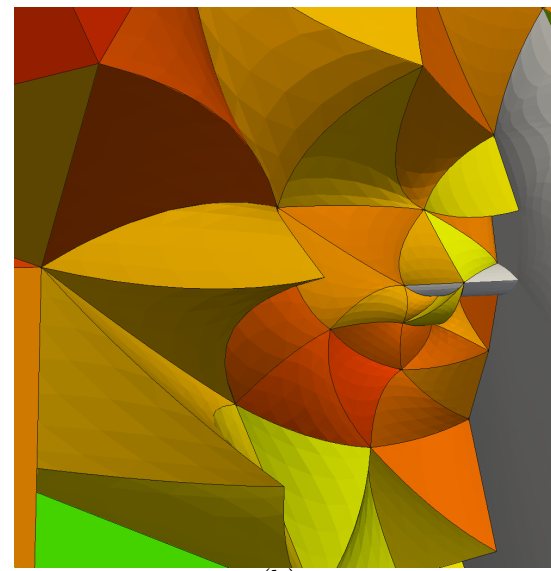

(b)

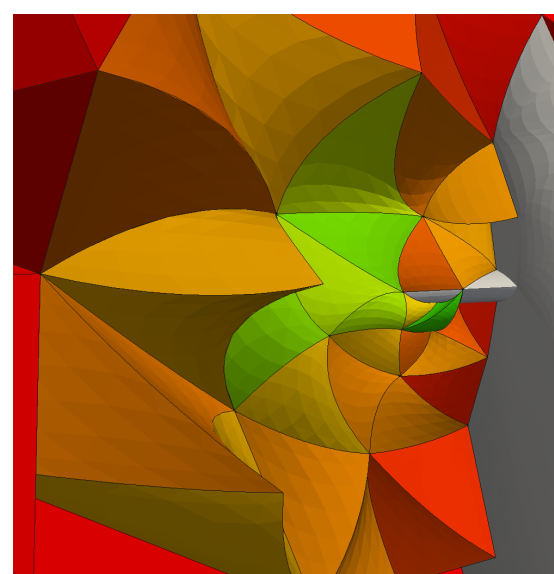

(d)

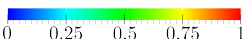

Fig. 9 Detail of the tetrahedral meshes of interpolation degree 4 for the exterior domain of a Falcon aircraft. (a,c) Initial meshes colored with respect to the equilateral and initial straight-sided ideals. (b,d) Smoothed meshes colored with respect to the equilateral and initial straight-sided ideals.

aircraft. That is, the proposed procedure has generated a high-order curved mesh composed of valid and high-quality elements that preserve the main geometrical features prescribed in the linear mesh.

\section{Concluding remarks}

In this work, we present a new technique to define distortion (quality) measures for high-order tetrahedral meshes. The proposed definition is valid for any interpolation degree and allows detecting the validity of a high-order tetrahedron. Moreover, the defined distortion measure can be used to generate 3D curved high- 
Table 4 Shape quality statistics of a mesh of interpolation degree 4 for a Falcon aircraft. The mesh is composed of 27511 elements and 317854 nodes.

\begin{tabular}{cccccccc}
\hline Mesh & Quality & Figure & Min. Q. & Max. Q. & Mean Q. & Std. Dev. & Tang. el. \\
\hline \multirow{2}{*}{ Initial } & Absolute & $7(\mathrm{~b}), 9(\mathrm{a})$ & 0.00 & 1.00 & 0.77 & 0.11 & 9 \\
& Relative & $9(\mathrm{c})$ & 0.00 & 1.00 & 0.99 & 0.02 & 9 \\
\multirow{2}{*}{ Smoothed } & Absolute & $7(\mathrm{~d}), 9(\mathrm{~b})$ & 0.21 & 1.00 & 0.78 & 0.11 & 0 \\
& Relative & $9(\mathrm{~d})$ & 0.32 & 1.00 & 0.99 & 0.04 & 0 \\
\hline
\end{tabular}

order meshes. Specifically, the distortion is capable of smoothing and untangling high-order meshes by means of an optimization procedure. Note that this is the final step of a curved meshing a posteriori approach. Moreover, we propose a $p$ continuation technique to accelerate the generation of curved high-order meshes. Finally, we show that the combination of the presented techniques allows the generation of valid curved high-order meshes from real CAD geometries.

Acknowledgements Work partially sponsored by the Spanish Ministerio de Ciencia e Innovación (grant DPI2011-23058), by the Ferran Sunyer i Balaguer Foundation (grant FSB 2013), and by CUR from DIUE of the Generalitat de Catalunya and the European Social Fund (grant FI-DGR).

\section{References}

1. Baart, M., Mulder, E.: A note on invertible two-dimensional quadratic finite element transformations. Commun. Appl. Numer. M. 3(6), 535-539 (1987)

2. Bassi, F., Rebay, S.: High-order accurate discontinuous finite element solution of the 2D Euler equations. J. Comput. Phys. 138(2), 251-285 (1997)

3. Branets, L., Carey, G.: Extension of a mesh quality metric for elements with a curved boundary edge or surface. J. Comput. Inf. Sci. Engrg. 5(4), 302-308 (2005)

4. Cantwell, C., Sherwin, S., Kirby, R., Kelly, P.: From $h$ to $p$ efficiently: selecting the optimal spectral/hp discretisation in three dimensions. Math. Model. Nat. Phenom. 6(3), 84-96 (2011)

5. Cantwell, C., Sherwin, S., Kirby, R., Kelly, P.: From $h$ to $p$ efficiently: strategy selection for operator evaluation on hexahedral and tetrahedral elements. Comput. Fluids 43(1), $23-28$ (2011)

6. Dey, S., O'Bara, R., Shephard, M.S.: Curvilinear mesh generation in 3D. Computer-Aided Design 33, 199-209 (2001)

7. Dey, S., Shephard, M.S., Flaherty, J.E.: Geometry representation issues associated with p-version finite element computations. Comput. Meth. Appl. M. 150(1-4), 39-55 (1997)

8. Escobar, J.M., Rodríguez, E., Montenegro, R., Montero, G., González-Yuste, J.M.: Simultaneous untangling and smoothing of tetrahedral meshes. Comput. Meth. Appl. Mech. Engrg. 192(25), 2775-2787 (2003)

9. Field, D.: Algorithms for determining invertible two-and three-dimensional quadratic isoparametric finite element transformations. Int. J. Numer. Meth. Engrg. 19(6), 789$802(1983)$

10. Field, D.: Qualitative measures for initial meshes. Int. J. Numer. Meth. Engrg. 47(4), 887-906 (2000)

11. Gargallo-Peiró, A.: Validation and generation of curved meshes for high-order unstructured methods. Ph.D. thesis, Universitat Politècnica de Catalunya (2014)

12. Gargallo-Peiró, A., Roca, X., Peraire, J., Sarrate, J.: Defining quality measures for mesh optimization on parameterized CAD surfaces. In: Proc. 21st Int. Meshing Roundtable, pp. 85-102. Springer International Publishing (2013)

13. Gargallo-Peiró, A., Roca, X., Peraire, J., Sarrate, J.: Defining quality measures for validation and generation of high-order tetrahedral meshes. In: Proc. 22nd Int. Meshing Roundtable, pp. 109-126. Springer International Publishing (2014) 
14. Gargallo-Peiró, A., Roca, X., Peraire, J., Sarrate, J.: Optimization of a regularized distortion measure to generate curved high-order unstructured tetrahedral meshes. Preprint (2014)

15. Gargallo-Peiró, A., Roca, X., Sarrate, J.: A surface mesh smoothing and untangling method independent of the CAD parameterization. Comput. Mech. 53(4), 587-609 (2014). DOI 10.1007/s00466-013-0920-1. URL http://dx.doi.org/10.1007/s00466-013-0920-1

16. George, P.L., Borouchaki, H.: Construction of tetrahedral meshes of degree two. Int J Numer Meth Eng 90(9), 1156-1182 (2012)

17. Hesthaven, J., Warburton, T.: Nodal Discontinuous Galerkin Methods: Algorithms, Analysis, and Applications. Texts in Applied Mathematics. Springer (2007). URL http: //books.google.es/books? id=APQkDOmwyksC

18. Huerta, A., Angeloski, A., Roca, X., Peraire, J.: Efficiency of high-order elements for continuous and discontinuous Galerkin methods. Int. J. Numer. Meth. Engrg. 96, 529560 (2013). DOI 10.1002/nme.4547

19. Huerta, A., Roca, X., Angeloski, A., Peraire, J.: Are high-order and hybridizable discontinuous Galerkin methods competitive? Oberwolfach Reports 9(1), 485 - 487 (2012)

20. Johnen, A., Remacle, J.F., Geuzaine, C.: Geometrical validity of curvilinear finite elements. In: Proc. 20th Int. Meshing Roundtable, pp. 255-271. Springer International Publishing (2012)

21. Johnen, A., Remacle, J.F., Geuzaine, C.: Geometrical validity of curvilinear finite elements. J. Comput. Phys. 233, $359-372$ (2013)

22. Kirby, R., Sherwin, S., Cockburn, B.: To CG or to HDG: a comparative study. J. Sci. Comput. 51(1), 183-212 (2012)

23. Knupp, P.M.: Algebraic mesh quality metrics. SIAM J. Numer. Anal. 23(1), 193-218 (2001)

24. Knupp, P.M.: Algebraic mesh quality metrics for unstructured initial meshes. Finite Elem. Anal. Des. 39(3), 217-241 (2003)

25. Knupp, P.M.: Label-invariant mesh quality metrics. In: Proc. 18th Int. Meshing Roundtable, pp. 139-155. Springer Berlin Heidelberg, Salt Lake City (2009)

26. Löhner, R.: Error and work estimates for high-order elements. Int. J. Numer. Meth. Fluids 67(12), 2184-2188 (2011)

27. Löhner, R.: Improved error and work estimates for high-order elements. Int. J. Numer. Meth. Fluids 72, 1207-1218 (2013)

28. Luo, X., Shephard, M.S., O'Bara, R., Nastasia, R., Beall, M.: Automatic p-version mesh generation for curved domains. Engrg. Comput. 20(3), 273-285 (2004)

29. Luo, X., Shephard, M.S., Remacle, J.F.: The influence of geometric approximation on the accuracy of higher order methods. In: 8th Int. Conf. Numerical Grid Generation in Computational Field Simulations (2002)

30. Luo, X., Shephard, M.S., Remacle, J.F., O'Bara, R., Beall, M., Szabó, B., Actis, R.: Pversion mesh generation issues. In: Proc. 11th Int. Meshing Roundtable, pp. 343-354. Springer Berlin Heidelberg (2002)

31. Mitchell, A., Phillips, G., Wachspress, E.: Forbidden shapes in the finite element method. IMA J. Appl. Math. 8(2), 260 (1971)

32. Persson, P.O., Peraire, J.: Curved mesh generation and mesh refinement using lagrangian solid mechanics. In: Proc. 47th AIAA (2009)

33. Remacle, J.F., Toulorge, T., Lambrechts, J.: Robust untangling of curvilinear meshes. In: Proc. 21st Int. Meshing Roundtable, pp. 71-83. Springer International Publishing (2013)

34. Roca, X., Gargallo-Peiró, A., Sarrate, J.: Defining quality measures for high-order planar triangles and curved mesh generation. In: Proc. 20th Int. Meshing Roundtable, pp. 365383. Springer International Publishing (2012)

35. Roca, X., Ruiz-Gironés, E., Sarrate, J.: EZ4U: Mesh generation environment. www-lacan. upc.edu/ez4u.htm (2010)

36. Roca, X., Sarrate, J., Ruiz-Gironés, E.: A graphical modeling and mesh generation environment for simulations based on boundary representation data. In: Communications in Num. Meth. Engrg. Porto (2007)

37. Salem, A., Canann, S., Saigal, S.: Robust distortion metric for quadratic triangular 2D finite elements. Appl. Mech. Div. ASME. 220, 73-80 (1997)

38. Salem, A., Canann, S., Saigal, S.: Mid-node admissible spaces for quadratic triangular arbitrarily curved 2D finite elements. Int. J. Numer. Meth. Engrg. 50(2), 253-272 (2001)

39. Salem, A., Saigal, S., Canann, S.: Mid-node admissible space for 3D quadratic tetrahedral finite elements. Engrg. Comput. 17(1), 39-54 (2001) 
40. Sastry, S., Shontz, S., Vavasis, S.: A log-barrier method for mesh quality improvement. In: Proc. 20th Int. Meshing Roundtable, pp. 329-346. Springer International Publishing (2012)

41. Sastry, S., Shontz, S., Vavasis, S.: A log-barrier method for mesh quality improvement and untangling. Engrg. Comput. (Published online ahead of print) (2012). DOI 10.1007/s00366-012-0294-6. URL http://dx.doi.org/10.1007/s00366-012-0294-6

42. Sevilla, R., Fernández-Méndez, S., Huerta, A.: NURBS-Enhanced Finite Element Method (NEFEM): a seamless bridge between CAD and FEM. Arch. Comput. Meth. Engrg. 18(4), 441-484 (2011)

43. Shephard, M.S., Flaherty, J.E., Jansen, K., Li, X., Luo, X., Chevaugeon, N., Remacle, J.F., Beall, M., O’Bara, R.: Adaptive mesh generation for curved domains. Appl. Numer. Math. 52(2-3), 251-271 (2005)

44. Sherwin, S., Peiró, J.: Mesh generation in curvilinear domains using high-order elements. Int. J. Numer. Meth. Engrg. 53(1), 207-223 (2002)

45. Shewchuk, J.: What is a good linear finite element? interpolation, conditioning, anisotropy, and quality measures. Preprint (2002)

46. Toulorge, T., Geuzaine, C., Remacle, J.F., Lambrechts, J.: Robust untangling of curvilinear meshes. J. Comput. Phys. 254, 8 - 26 (2013)

47. Vos, P.E., Sherwin, S., Kirby, R.: From $h$ to $p$ efficiently: implementing finite and spectral $/ h p$ element methods to achieve optimal performance for low- and high-order discretisations. J. Comput. Phys. 229(13), 5161-5181 (2010)

48. Xie, Z., Sevilla, R., Hassan, O., Morgan, K.: The generation of arbitrary order curved meshes for 3D finite element analysis. Comput. Mech. 51, 361-374 (2012)

49. Xue, D., Demkowicz, L.: Control of geometry induced error in $h p$ finite element (FE) simulations. I. Evaluation of FE error for curvilinear geometries. Internat. J. Numer. Anal. Model. 2(3), 283-300 (2005)

50. Yuan, K., Huang, Y., Pian, T.: Inverse mapping and distortion measures for quadrilaterals with curved boundaries. Int. J. Numer. Meth. Engrg. 37(5), 861-875 (1994) 